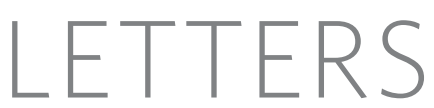

\title{
Origins and evolutionary genomics of the 2009 swine-origin H1N1 influenza A epidemic
}

\author{
Gavin J. D. Smith ${ }^{1}$, Dhanasekaran Vijaykrishna ${ }^{1}$, Justin Bahl ${ }^{1}$, Samantha J. Lycett ${ }^{2}$, Michael Worobey ${ }^{3}$, \\ Oliver G. Pybus ${ }^{4}$, Siu Kit Ma ${ }^{1}$, Chung Lam Cheung ${ }^{1}$, Jayna Raghwani ${ }^{2}$, Samir Bhatt ${ }^{4}$, J. S. Malik Peiris ${ }^{1}$, Yi Guan $^{1}$ \\ $\&$ Andrew Rambaut ${ }^{2}$
}

In March and early April 2009, a new swine-origin influenza A (H1N1) virus (S-OIV) emerged in Mexico and the United States ${ }^{1}$. During the first few weeks of surveillance, the virus spread worldwide to 30 countries (as of May 11) by human-to-human transmission, causing the World Health Organization to raise its pandemic alert to level 5 of 6 . This virus has the potential to develop into the first influenza pandemic of the twenty-first century. Here we use evolutionary analysis to estimate the timescale of the origins and the early development of the S-OIV epidemic. We show that it was derived from several viruses circulating in swine, and that the initial transmission to humans occurred several months before recognition of the outbreak. A phylogenetic estimate of the gaps in genetic surveillance indicates a long period of unsampled ancestry before the S-OIV outbreak, suggesting that the reassortment of swine lineages may have occurred years before emergence in humans, and that the multiple genetic ancestry of S-OIV is not indicative of an artificial origin. Furthermore, the unsampled history of the epidemic means that the nature and location of the genetically closest swine viruses reveal little about the immediate origin of the epidemic, despite the fact that we included a panel of closely related and previously unpublished swine influenza isolates. Our results highlight the need for systematic surveillance of influenza in swine, and provide evidence that the mixing of new genetic elements in swine can result in the emergence of viruses with pandemic potential in humans ${ }^{2}$.

Initial genetic characterization of the S-OIV outbreak by the United States Centers for Disease Control suggested swine as its probable source, on the basis of sequence similarity to previously reported swine influenza isolates ${ }^{1}$. Classical swine $\mathrm{H} 1 \mathrm{~N} 1$ viruses have circulated in pigs in North America and other regions for at least 80 years $^{3}$. In 1998, a new triple-reassortant H3N2 virus-comprising genes from classical swine H1N1, North American avian, and human H3N2 (A/Sydney/5/97-like) influenza-was reported as the cause of outbreaks in North American swine, with subsequent establishment in pig populations $s^{4,5}$. Co-circulation and mixing of the triple-reassortant $\mathrm{H} 3 \mathrm{~N} 2$ with established swine lineages subsequently generated further H1N1 and H1N2 reassortant swine viruses ${ }^{6-8}$, which have caused sporadic human infections in the United States since 2005 (refs 6, 7). Consequently, human infection with H1N1 swine influenza has been a nationally notifiable disease in the United States since 2007 (ref. 9). In Europe, an avian $\mathrm{H} 1 \mathrm{~N} 1$ virus was introduced to pigs ('avian-like' swine H1N1) and first detected in Belgium in 1979 (ref. 10). This lineage became established and gradually replaced classical swine $\mathrm{H} 1 \mathrm{~N} 1$ viruses, and also reassorted in pigs with human
H3N2 viruses (A/Port Chalmers/1/1973-like) ${ }^{11}$. It is noteworthy that, until now, there has been no evidence of Eurasian avian-like swine $\mathrm{H} 1 \mathrm{~N} 1$ circulating in North American pigs. In Asia, the classical swine influenza lineage circulates, in addition to other identified viruses, including human $\mathrm{H} 3 \mathrm{~N} 2$, Eurasian avian-like H1N1, and North American triple-reassortant H3N2 (refs 12, 13).

Using comprehensive phylogenetic analyses, we have estimated a temporal reconstruction of the complex reassortment history of the S-OIV outbreak, summarized in Fig. 1 (Methods). Our analyses showed that each segment of the S-OIV genome was nested within a well-established swine influenza lineage (that is, a lineage circulating primarily in swine for $>10$ years before the current outbreak). The most parsimonious interpretation of these results is therefore that the progenitor of the S-OIV epidemic originated in pigs. Some transmission of swine influenza has, however, been observed in secondary hosts in North America, for example, in turkeys ${ }^{14}$. Although the precise evolutionary pathway of the genesis of S-OIV is greatly hindered by the lack of surveillance data (see later), we can conclude that the polymerase genes, plus HA, NP and NS, emerged from a triplereassortant virus circulating in North American swine. The source triple-reassortant itself comprised genes derived from avian (PB2 and PA), human H3N2 (PB1) and classical swine (HA, NP and NS) lineages. In contrast, the NA and $\mathrm{M}$ gene segments have their origin in the Eurasian avian-like swine H1N1 lineage. Phylogenetic analyses from the early days of the outbreak, on the basis of the first publicly available sequences, quickly established this multiple genetic origin (refs 8, 15, 16 and http://influenza.bio.ed.ac.uk).

Given that S-OIV contains genes of Eurasian origin, we included in our phylogenetic analyses 15 newly sequenced swine influenza viruses from Hong Kong, sampled in the course of a surveillance program conducted since the early 1990s. The viruses were a mixture of seven $\mathrm{H} 1 \mathrm{~N} 1$ and eight H1N2 subtypes, and viruses belonging to the classical, Eurasian avian-like, and triple-reassortant swine lineages were all present. Both Eurasian and triple-reassortant strains were isolated in Hong Kong in 2009. Extensive reassortment among these three virus lineages was also observed from the Hong Kong surveillance data (Supplementary Table 3), with reassortment between Eurasian avian-like and triple-reassortant swine lineages occurring as early as 2003 (for example, Sw/HK/78/2003).

Notably, for the PB1, HA and M genes, some of these newly generated sequences are more similar to the S-OIV epidemic than any previously reported isolates (Supplementary Fig. 2). Notably, seven out of eight genomic segments found in a single 2004 isolate (Sw/HK/915/04 (H1N2)) were located in a sister lineage to the current outbreak. Not only does this suggest that the precursors of S-OIV were swine viruses, 


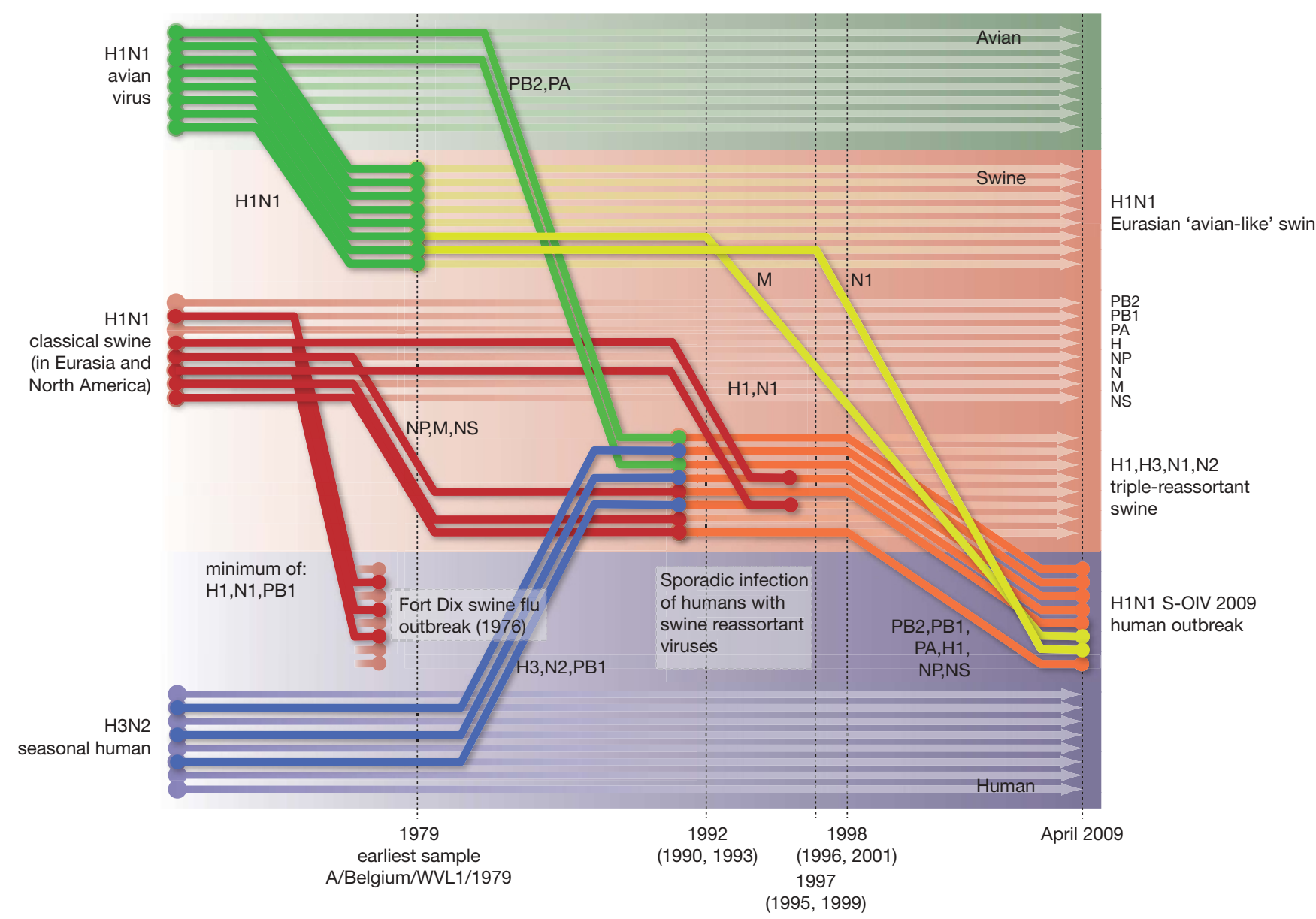

Figure 1 Reconstruction of the sequence of reassortment events leading up to the emergence of S-OIV. Shaded boxes represent host species; avian (green), swine (red) and human (grey). Coloured lines represent interspecies-transmission pathways of influenza genes. The eight genomic segments are represented as parallel lines in descending order of size. Dates marked with dashed vertical lines on 'elbows' indicate the mean time of

but also that they were geographically widely distributed. Crucially, however, the observation of a sister relationship between the current outbreak virus and Sw/HK/915/04 cannot be interpreted as evidence for a Eurasian origin of the outbreak, owing to the long branch of the phylogeny leading to the 2009 human strains (Fig. 2 and Table 1). This branch must represent either an increased rate of evolution leading to the outbreak, or a long period during which the ancestors of the current epidemic went unsampled. To test these hypotheses, we regressed genetic divergence against sampling date for each gene, and found in favour of the latter: the evolutionary rate preceding the S-OIV epidemic is entirely typical for swine influenza (Supplementary Figs 2 and 3 ).

Therefore, to quantify the period of unsampled diversity, and to estimate the date of origin for the S-OIV outbreak, we performed a Bayesian molecular clock analysis for each gene (Methods). We also estimated the rate of evolution and time of the most recent common ancestor (TMRCA) of a set of genome sequences sampled from the S-OIV epidemic (between March and May 2009; isolates listed in Supplementary Table 4). We found that the common ancestor of the S-OIV outbreak and the closest related swine viruses existed between 9.2 and 17.2 years ago, depending on the genomic segment, hence the ancestors of the epidemic have been circulating undetected for about a decade. In contrast, the currently sampled S-OIV shared a common ancestor around January 2009 (no earlier than August 2008; Table 1). The long, unsampled history observed for every segment suggests that the reassortment of Eurasian and North American swine lineages may not have occurred recently, and it is possible that divergence of the S-OIV genes from corresponding virus lineages. Reassortment events not involved with the emergence of human disease are omitted. Fort Dix refers to the last major outbreak of S-OIV in humans. The first triple-reassortant swine viruses were detected in 1998, but to improve clarity the origin of this lineage is placed earlier.

this single reassortant lineage has been cryptically circulating rather than two distinct lineages of swine flu. Thus, this genomic structure may have been circulating in pigs for several years before emergence in humans, and we urge caution in making inferences about human adaptation on the basis of the ancestry of the individual genes.

A search for amino acid residues in the S-OIV outbreak sequences that have been previously identified as phenotypic markers showed no evidence of virulence-associated variation or adaptations to human hosts ${ }^{17-19}$, consistent with the outbreak being of swine origin and causing relatively mild symptoms. Full molecular characterization of the human swine H1N1 viruses is provided in Supplementary Information.

We did detect a difference in the viral molecular evolution in the outbreak clade when compared to that observed in related swine influenza sequences: all S-OIV genes showed a comparatively higher non-synonymous to synonymous $\left(d_{\mathrm{N}} / d_{\mathrm{S}}\right)$ substitution rate ratio (Supplementary Tables 1 and 2). This $d_{\mathrm{N}} / d_{\mathrm{S}}$ ratio rise could be due to the increased detection of mildly deleterious mutations resulting from intensive epidemic surveillance; such mutations would more typically be eliminated and escape detection ${ }^{20}$. Alternatively, these mutations could be adaptations to the new host species.

Because this $d_{\mathrm{N}} / d_{\mathrm{S}}$ ratio rise may affect our estimate of the TMRCA of the S-OIV outbreak strains (which was estimated using long-term rates of swine influenza evolution), we compared the mean $d_{\mathrm{N}} / d_{\mathrm{S}}$ values of outbreak versus non-outbreak data sets, thereby approximating the degree of excess of non-synonymous mutations in the outbreak sequences (Methods). Once the $d_{\mathrm{N}} / d_{\mathrm{S}}$ ratio rise is corrected 

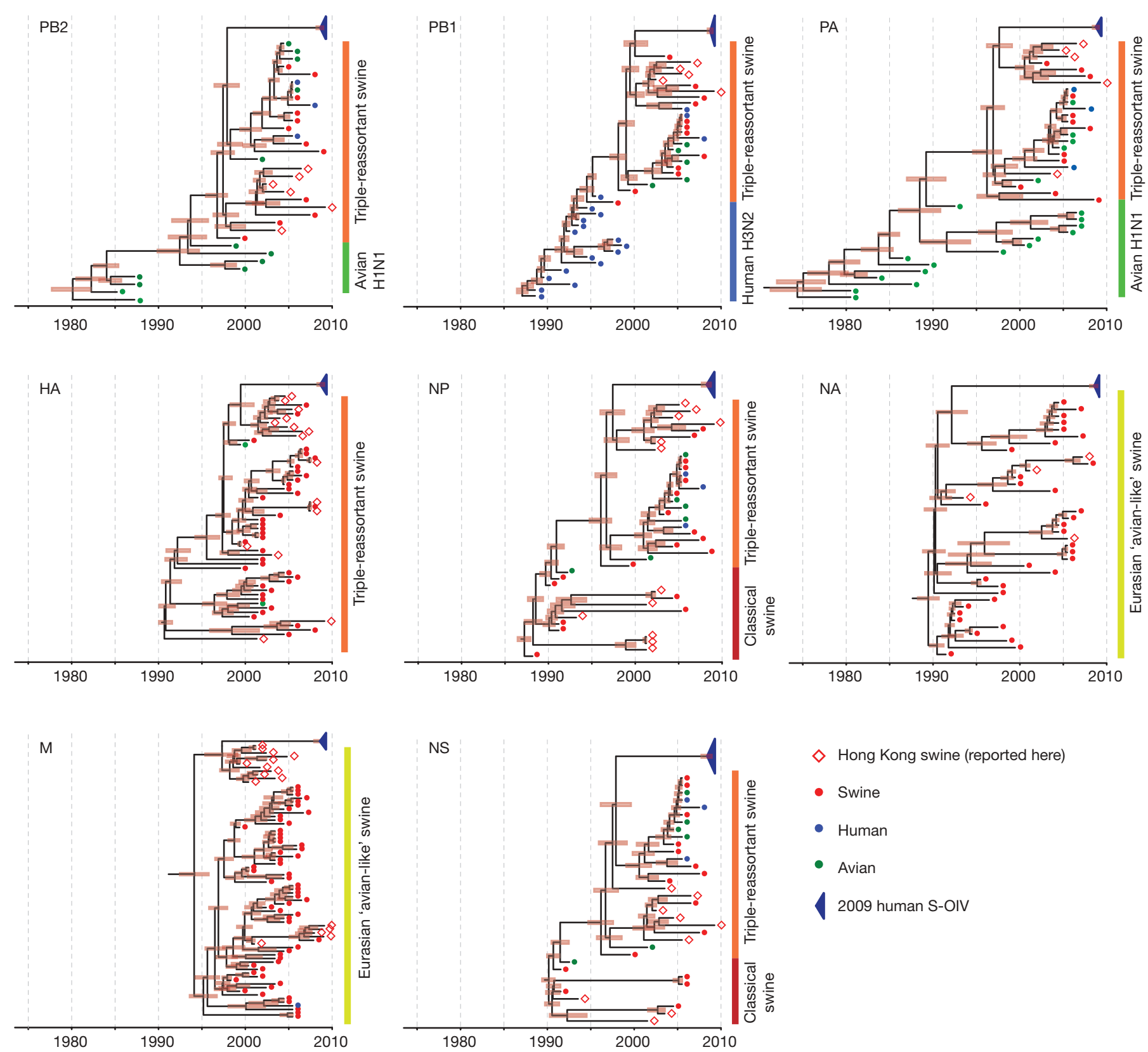

$\diamond$ Hong Kong swine (reported here)

- Swine

- Human

- Avian

2009 human S-OIV

Figure 2 | Genetic relationships and timing of S-OIV for each genomic segment. Symbols represent sampled viruses on a timescale of when they were sampled and coloured by host species (pigs, red; humans, blue; birds, green). Internal nodes are reconstructed common ancestors with $95 \%$

for, the mean TMRCA of the S-OIV outbreak became 1 to 5 months more recent for each gene (Supplementary Tables 1 and 2). Furthermore, the adjusted TMRCA estimates are more uniform across genes, and are more similar to that obtained using internally credible intervals on their date given by the red bars. The S-OIV outbreak strains are represented by a blue triangle, with the apex representing the common ancestor of these.

calibrated S-OIV complete genomes (Table 1; a comparable estimate for the TMRCA of the HA gene only was recently reported ${ }^{21}$ ). Irrespective of whether the $d_{\mathrm{N}} / d_{\mathrm{S}}$ ratio rise is due to increased detection of deleterious mutations or to increased adaptive evolution, its

Table 1 | Time of most recent common ancestors for the S-OIV outbreak

\begin{tabular}{llll}
\hline Gene & TMRCA of outbreak samples & Duration of unsampled diversity (years) & Mean evolutionary rate $\times 10^{-3}$ (subst. per site per year) \\
\hline HA & 28 Aug 2008 (1 Apr 2008, 2 Jan 2009) & $9.80(8.41,11.02)$ & $3.67(3.41,3.92)$ \\
MP & 3 Aug 2008 (8 Dec 2007, 5 Feb 2009) & $11.82(10.17,13.74)$ & $2.55(2.19,2.93)$ \\
NA & 8 Aug 2008 (23 Feb 2008, 26 Dec 2008) & $17.15(15.40,18.88)$ & $3.65(3.22,4.12)$ \\
NP & 27 Mar 2008 (15 Sep 2007, 19 Sep 2008) & $11.83(10.53,13.23)$ & $2.59(2.34,2.84)$ \\
NS & 21 May 2008 (30 Sep 2007, 27 Nov 2008) & $11.47(9.75,13.21)$ & $2.62(2.32,2.92)$ \\
PA & 7 Oct 2008 (1 Jun 2008, 1 Feb 2009) & $11.70(10.25,13.10)$ & $2.45(2.20,2.69)$ \\
PB1 & 24 Oct 2008 (8 Jul 2008, 25 Jan 2009) & $9.24(7.59,10.48)$ & $2.34(2.13,2.53)$ \\
PB2 & 9 Sep 2008 (12 Apr 2008, 9 Jan 2009) & $11.26(9.93,12.69)$ & $2.60(2.29,2.92)$ \\
Genome* & 21 Jan 2009 (3 Aug 2008, 13 Mar 2009) & N/A & $3.66(0.61,6.58)$
\end{tabular}

The values in parentheses represent the $95 \%$ credible intervals.

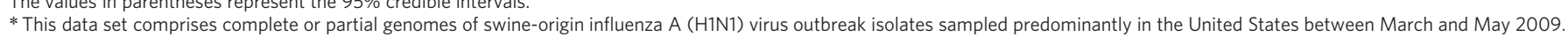


presence may be a general feature of intensively sampled emerging epidemics, and should be accounted for in the evolutionary analysis of such events.

Movement of live pigs between Eurasia and North America seems to have facilitated the mixing of diverse swine influenza viruses, leading to the multiple reassortment events associated with the genesis of the S-OIV strain. Domestic pigs have been described as a hypothetical 'mixing-vessel', mediating by reassortment the emergence of new influenza viruses with avian or avian-like genes into the human population, and triggering a pandemic associated with antigenic shift ${ }^{2}$. Previous research has suggested that occupational exposure to pigs increases the risk of swine influenza virus infection, and that swine workers should be considered in any surveillance programs ${ }^{22}$.

The emergence of S-OIV provides further evidence of the role of domestic pigs in the ecosystem of influenza A. As reported recently, all three pandemics of the twentieth century seem to have been generated by a series of multiple reassortment events in swine or humans, and to have emerged over a period of years before pandemic recognition ${ }^{23}$. Our results show that the genesis of the S-OIV epidemic followed a similar evolutionary pathway: H1N1 viruses with human pandemic potential had been identified, transmission from swine to humans was known $^{5}$ and the disease had been made notifiable. Yet despite widespread influenza surveillance in humans, the lack of systematic swine surveillance allowed for the undetected persistence and evolution of this potentially pandemic strain for many years.

\section{METHODS SUMMARY}

We compared 15 newly sequenced Hong Kong swine influenza genomes and two genomes from the S-OIV outbreak with 796 genomes representing the spectrum of influenza A diversity (comprising 285 human, 100 swine and 411 avian isolates). Phylogenetic trees were constructed for each genomic segment independently (Supplementary Fig. 1). Next, for each genomic segment, viruses with known isolation dates that were genetically similar to the current outbreak were identified, and more detailed analysis using a Bayesian 'relaxed molecular clock' approach was performed ${ }^{24}$, thereby estimating rates of viral evolution and dates of divergence (Fig. 2). Finally, a similar Bayesian molecular clock approach was applied to the 30 individual viruses isolated from the human outbreak since the end of March 2009 (Supplementary Table 4 and Supplementary Fig. 2). This analysis was performed assuming a model of exponential growth in the number of infections.

Full Methods and any associated references are available in the online version of the paper at www.nature.com/nature.

Received 24 May; accepted 4 June 2009.

Published online 11 June 2009.

1. Centers for Disease Control and Prevention. Swine influenza A (H1N1) infection in two children-Southern California, March-April 2009. Morb. Mortal. Wkly Rep. $58,400-402$ (2009).

2. Shortridge, K. F., Webster, R. G., Butterfield, W. K. \& Campbell, C. H. Persistence of Hong Kong influenza virus variants in pigs. Science 196, 1454-1455 (1977).

3. Shope, R. E. \& Lewis, P. Swine influenza: experimental transmission and pathology. J. Exp. Med. 54, 349-359 (1931).

4. Brown, I. H., Harris, P. A., McCauley, J. W. \& Alexander, D. J. Multiple genetic reassortment of avian and human influenza $A$ viruses in European pigs, resulting in the emergence of an H1N2 virus of novel genotype. J. Gen. Virol. 79, 2947-2955 (1998).

5. Webby, R. J. et al. Evolution of swine H3N2 influenza viruses in the United States. J. Virol. 74, 8243-8251 (2000).
6. Newman, A. P. et al. Human case of swine influenza A ( $\mathrm{H} 1 \mathrm{~N} 1)$ triple reassortant virus infection, Wisconsin. Emerg. Infect. Dis. 14, 1470-1472 (2008).

7. Shinde, V. et al. Triple-reassortant swine influenza A ( $\mathrm{H} 1)$ in humans in the United States, 2005-2009. N. Engl. J. Med. doi:10.1056/NEJMoa0903812 (in the press).

8. Novel Swine-Origin Influenza A (H1N1) Virus Investigation Team. Emergence of a novel swine-origin influenza A (H1N1) virus in humans. N. Engl. J. Med. doi:10.1056/NEJMoa0903810 (in the press).

9. Centers for Disease Control and Prevention. Novel influenza A virus infections2007 case definition. <http://www.cdc.gov/ncphi/disss/nndss/casedef/ novel_influenzaA.htm> (24 May 2009).

10. Pensaert, M., Ottis, K., Vanderputte, J., Kaplan, M. M. \& Buchmann, P. A. Evidence for the natural transmission of influenza $A$ virus from wild ducks to swine and its potential for man. Bull. World Health Organ. 59, 75-78 (1981).

11. Brown, I. H. The epidemiology and evolution of influenza viruses in pigs. Vet. Microbiol. 74, 29-46 (2000)

12. Peiris, J. S. M. et al. Cocirculation of avian H9N2 and contemporary "human" H3N2 influenza A viruses in pigs in southeastern China: potential for genetic reassortment? J. Virol. 75, 9679-9686 (2001).

13. Jung, K. \& Song, D. S. Evidence of the cocirculation of influenza H1N1, H1N2 and H3N2 viruses in the pig population of Korea. Vet. Rec. 161, 104-105 (2007).

14. Choi, Y. K. et al. H3N2 influenza virus transmission from swine to turkeys, United States. Emerg. Infect. Dis. 10, 2156-2160 (2004).

15. Trifonov, V., Khiabanian, H., Greenbaum, B. \& Rabadan, R. The origin of the recent swine influenza A (H1N1) virus infecting humans. Euro Surveill. 14, 19193 (2009).

16. Garten, R. J. et al. Antigenic and genetic characteristics of swine-origin 2009 A (H1N1) influenza viruses circulating in humans. Science doi:10.1126/ science.1176225 (in the press).

17. Hatta, M., Gao, P., Halfmann, P. \& Kawaoka, Y. Molecular basis of high virulence of Hong Kong H5N1 influenza A viruses. Science 7, 1840-1842 (2001).

18. Le, Q. M. Sakai-Tagawa, Y., Ozawa, M., Ito, M. \& Kawaoka, Y. Selection of H5N1 influenza virus PB2 during replication in humans. J. Virol. 83, 5278-5281 (2009).

19. Obenauer, J. C. et al. Large-scale sequence analysis of avian influenza isolates. Science 311, 1576-1580 (2006)

20. Pybus, O. G. et al. Phylogenetic estimation of deleterious mutation load in RNA viruses and its contribution to viral evolution. Mol. Biol. Evol. 24, 845-852 (2007).

21. Fraser, C. et al. Pandemic potential of a strain of influenza A (H1N1): Early findings. Science doi:10.1126/science.1176062 (in the press).

22. Myers, K. P. et al. Are swine workers in the United States at increased risk of infection with zoonotic influenza virus? Clin. Infect. Dis. 42, 14-20 (2006).

23. Smith, G. J. D. et al. Dating the emergence of pandemic influenza viruses. Proc. Natl Acad. Sci. USA. (in the press).

24. Drummond, A. J. \& Rambaut, A. BEAST: Bayesian evolutionary analysis by sampling trees. BMC Evol. Biol. 7, 214 (2007).

Supplementary Information is linked to the online version of the paper at www.nature.com/nature.

Acknowledgements We thank E. C. Holmes for comments and encouragement. We acknowledge support from The Royal Society of London (A.R. and O.G.P.), the National Institute of Allergy and Infectious Diseases (NIAID) (G.J.D.S. and M.W.), the Biotechnology and Biological Sciences Research Council (BBSRC) (S.J.L.), and the David and Lucile Packard Foundation (M.W.). A.R. works as a part of the Interdisciplinary Centre for Human and Avian Influenza Research (ICHAIR). This study was supported by the National Institutes of Health (NIAID contract HHSN266200700005C) and the Area of Excellence Scheme of the University Grants Committee (grant AoE/M-12/06) of the Hong Kong SAR Government.

Author Contributions J.B., S.J.L., O.G.P., A.R., G.J.D.S., D.V. and M.W. conceived the study, performed analyses, co-wrote the paper, and all contributed equally to this work. J.S.M.P. co-wrote the paper, Y.G. conceived the study and co-wrote the paper, S.B. and J.R. performed analyses, S.K.M. conducted surveillance, and C.L.C. conducted sequencing. All authors commented on and edited the paper.

Author Information Newly reported sequences have been deposited at GenBank under accession numbers GQ229259-GQ229378. Reprints and permissions information is available at www.nature.com/reprints. This paper is distributed under the terms of the Creative Commons Attribution-Non-Commercial-Share Alike licence, and is freely available to all readers at www.nature.com/nature. Correspondence and requests for materials should be addressed to A.R. (a.rambaut@ed.ac.uk) or Y.G. (yguan@hku.hk). 


\section{METHODS}

Sequence selection for phylogenetic analysis. We downloaded 3,986 complete influenza genomes of any subtype and sampling year (2,490 human, 185 swine and 1,311 avian) from the NCBI Influenza Virus Resource ${ }^{25}$ on 29 April 2009. Each sequence set was given a unique ID of the form (ID number)_(Subtype) (Host)_(isolate name), in which the isolate name is in lower case.

To reduce the number of very similar sequences, we listed all isolates in which the coding region in segment 1 (PB2) was at least one nucleotide different from the others. This left 1,759 human, 166 swine and 1,117 avian complete genome sets. Next we sampled the human, swine and avian sets, selecting one genome set per specific host (as defined in the isolate name, for example, chicken, duck), per specific location (for example, state or province), per year (although isolate name synonyms, for example, duck $=\mathrm{dk}$, hongkong $=\mathrm{hk}$ were not accounted for). Two avian and four swine sequence sets were removed owing to bad sequences in one or more segments (for example, frameshifts), leaving 286 human (including S-OIVs), 100 swine and 411 avian sequences in the sampled subset. A further outbreak sequence set (A/Canada-ON/RV1527/2009), and the 15 new swine sequence sets were also added, making a total of 813 complete genome sets for analysis. For the more detailed, temporal analyses, all available S-OIV sequences were used.

The nucleotides in the coding regions of segments 1 (PB2), 2 (PB1), 3 (PA) and 5 (NP) were aligned using ClustalW ${ }^{26}$ followed by manual alignment to codon position. The full nucleotide sequences of segments 7 (M1 and M2) and 8 (NS1 and NS2) were also aligned using ClustalW, and the sequences were edited such that all of the codons in first open reading frame (ORF) were followed by the remaining codons in the second ORF (that is, nucleotides were not repeated between the two ORFs). The HA and NA genes (segments 4 and 6) were aligned to codon positions using Muscle ${ }^{27}$. Further $\mathrm{H} 1, \mathrm{H} 3, \mathrm{~N} 1$ and N2 only alignments were also performed.

New swine influenza sequences from Hong Kong. To evaluate the evolutionary history of swine/human influenza A H1N1 viruses, 15 viruses isolated from swine in Hong Kong during 1993 to 2009 were sequenced. Viral RNA was directly extracted from infected allantoic fluid or cell culture using QIAamp viral RNA minikit (Qiagen, Inc.). Complementary DNA was synthesized by reverse transcription reaction, and gene amplification by PCR was performed using specific primers for each gene segment. PCR products were purified with the QIAquick PCR purification kit (Qiagen Inc.) and sequenced by synthetic oligonucleotides. Reactions were performed using Big Dye-Terminator v3.1 Cycle Sequencing Reaction Kit on an ABI PRISM 3730 DNA Analyser (Applied Biosystems) following the manufacturer's instructions. All sequences were assembled and edited with Lasergene version 8.0 (DNASTAR). Full genome sequences of these viruses are available for download at GenBank under accession numbers GQ229259-GQ229378.

Molecular evolution and adaptation. We used the programs SLAC (SingleLikelihood Ancestor Counting) ${ }^{28}$ and SNAP (Synonymous Non-synonymous Analysis Program $)^{29}$ to compare the mean ratio of non-synonymous changes per non-synonymous site to synonymous changes per synonymous site $\left(d_{\mathrm{N}} / d_{\mathrm{S}}\right)$ of outbreak versus non-outbreak sequences. SLAC calculates inferred ancestral sequences for each internal node in a phylogeny using a codon model (and disallowing stop codons), and then counts the synonymous and non-synonymous mutations by comparing each codon to its immediate ancestor. SNAP counts the possible synonymous and non-synonymous codon changes across all pairs of sequences.

In brief, we calculated the effect of the excess of non-synonymous changes in the outbreak data as follows. Assume that $S$ is the number of synonymous sites in a data set, $N$ is the number of non-synonymous sites (typically $\sim 3.5 S$ for these data), and $\omega$ is the $d_{\mathrm{N}} / d_{\mathrm{S}}$ ratio. If the proportional contribution to the overall rate from synonymous sites is $s$, then the proportional contribution to the overall rate from non-synonymous sites is equal to $(N / S)(\omega) s . N, S$ and $\omega$ are all readily estimated from the data. Assuming the same rate of synonymous substitution in both the outbreak and reference data sets, the relative rate expected in the outbreak sequences compared to the reference sequences is thus equal to

$$
\left(s+(N / S)\left(\omega_{\text {outbreak }}\right) s\right) /\left(s+(N / S)\left(\omega_{\text {reference }}\right) s\right)
$$

Phylogenetic analyses. Phylogenetic trees were inferred using the neighbourjoining distance method, with genetic distances calculated by maximum likelihood under the Hasegawa-Kishino-Yano (HKY) model with gamma-distributed rates among sites $(\mathrm{HKY}+\Gamma)$. Parameters of this model were estimated using maximum likelihood on an initial tree. Temporal phylogenies and rates of evolution were inferred using a relaxed molecular clock model that allows rates to vary among lineages within a Bayesian Markov chain Monte Carlo (MCMC) framework ${ }^{24}$. This was used to sample phylogenies and the dates of divergences between viruses from their joint posterior distribution, in which the sequences are constrained by their known date of sampling. A model comprising a codon-position-specific $\mathrm{HKY}+\Gamma$ substitution model was used. The limited sampling timespan of the S-OIV samples required a simpler model to avoid over-parameterization, so a single $\mathrm{HKY}+\Gamma$ model over all sites was used. For the analyses using Bayesian MCMC sampling, in all cases chain lengths of at least 50 million steps were used with a 10\% 'burn-in' removed. Furthermore, at least two independent runs of each were performed and compared to ensure adequate sampling.

25. Bao, Y. et al. The influenza virus resource at the national center for biotechnology information. J. Virol. 82, 596-601 (2008).

26. Thompson, J. D., Higgins, D. G. \& Gibson, T. J. CLUSTAL W: improving the sensitivity of progressive multiple sequence alignment through sequence weighting, position-specific gap penalties and weight matrix choice. Nucleic Acids Res. 22, 4673-4680 (1994).

27. Edgar, R. C. MUSCLE: multiple sequence alignment with high accuracy and high throughput. Nucleic Acids Res. 32, 1792-1797 (2004).

28. Kosakovsky Pond, S. L. \& Frost, S. D. W. Not so different after all: a comparison of methods for detecting amino acid sites under selection. Mol. Biol. Evol. 22, 1208-1222 (2005)

29. Korber, B. HIV Signature and Sequence Variation Analysis. Computational Analysis of HIV Molecular Sequences (eds Rodrigo, A. G. \& Learn, G. H.) Ch. 4, 55-72 (Kluwer Academic Publishers, 2000). 